\title{
Multi-Source Phase Retrieval from Multi-Channel Phaseless STFT Measurements
}

\author{
Yina Guo ${ }^{\mathrm{a}, *}$, Anhong Wang ${ }^{\mathrm{a}}$, Wenwu Wang ${ }^{\mathrm{b}, *}$ \\ ${ }^{a}$ Dept. of Electronics and Information Engineering, Taiyuan University \\ of Science and Technology, China \\ ${ }^{b}$ Centre for Vision, Speech and Signal Processing, University of Surrey, UK
}

\begin{abstract}
In a recent study, it was shown that, given only the magnitude of the short-time Fourier transform (STFT) of a signal, it is possible to recover the phase information of its STFT under certain conditions. However, this is only investigated for the single-source scenario. In this paper, we extend this work and formulate a multi-source phase retrieval problem where multi-channel phaseless STFT measurements are given as input. We then present a robust multi-source phase retrieval (RMSPR) algorithm based on a gradient descent (GD) algorithm by minimizing a non-convex loss function and independent component analysis (ICA). An improved least squares (LS) loss function is presented to find the initialization of the GD algorithm. Experimental evaluation has been conducted to show that under appropriate conditions the proposed algorithm can explicitly recover the phase of the sources, the mixing matrix, and the sources simultaneously, from noisy measurements.
\end{abstract}

Keywords: Multi-source phase retrieval, short-time Fourier transform (STFT), non-convex optimization, independent component analysis (ICA)

\section{Introduction}

Phase retrieval is referred to as the problem of recovering phase information from its Fourier transform magnitude. It is of paramount importance in various engineering and scientific applications, such as speech recognition [1], blind channel estimation

5 [2], X-ray crystallography [3], optics [4], and astronomy [5]. Many studies have been conducted to address this problem, of which the two main approaches are built upon sparsity prior knowledge [6], [7], [8] and additional measurements [9], [10], [1T] respectively. Phase retrieval from the STFT measurement is among the latter approach that has received increasing interest recently [112], [13] and is the focus of this paper.

Phase retrieval algorithms are often designed for a single source, such as the recent work by Bendory and Eldar [13] which was proposed for extracting the phase information from the phaseless STFT measurements of a single source. However, in some

*Corresponding author. Email address: zulibest@163.com,w.wang@ @urrey.ac.uk 
applications such as the shaped-beam synthesis of antenna arrays [14], CCD cameras and photosensitive films [15], the measurements may be a mixture (or mixtures) of multiple sources. This is an inherently ill-posed problem due to the lack of the phase and the mixing information, and the existing single-source based phase retrieval algorithms are not explicitly designed for this case.

In this paper, we extend the study in [13] to a multi-source scenario. Our algorithm differs from [13] in three important aspects. First, a new model is formed for multi-

20 source phase retrieval problem. Second, we present an algorithm which couples the ICA method with a gradient descent (GD) algorithm by minimizing a non-convex loss function. Third, the GD algorithm depends heavily on the initialization method and the geometry of the loss function, therefore we also present a modified least-square (LS) loss function to improve the initialization of the GD algorithm.

This paper is organized as follows. Section II describes the background. Section III formulates a mathematical model and an algorithm for the problem of multi-source phase retrieval. Section IV shows numerical experiments and results. Section V concludes the paper and draws potential future research directions.

Notation: Boldface small and capital letters denote vectors and matrices, respecso tively. The superscript ' $T$, ', $*$ ' and ' $\dagger$ ' denote the transpose, Hermitian and MoorePenrose pseudo-inverse of a matrix. $\lceil\cdot\rceil$ rounds the argument to the smallest integer that is not less than the argument. We use ' $\circ$ ' for the Hadamard (point-wise) product. $\operatorname{tr}(\cdot)$ takes the trace of a matrix. The $l$ th circular diagonal element of a matrix is denoted by $\operatorname{diag}(\cdot, l) . \mathrm{k}(\cdot)$ and $\operatorname{Off}(\cdot)$ represent the kurtosis and the off-diagonal element of its

35 argument.

\section{Background}

This section presents a brief overview of the method in [13].

The STFT of a 1D signal $\mathbf{x} \in \mathbb{C}^{N}$ is defined as the Fourier transform of the signal multiplied by a real sliding window $g$ of length $2 \leq W \leq N$.

$$
X(\tau, k)=\sum_{n=0}^{N-1} x(n) g(\tau L-n) e^{-2 j \pi k n / N},
$$

where $L$ is the maximal overlap between adjacent windows, $R=\lceil N / L\rceil$ denotes the number of short-time sections considered, $\tau=0, \ldots, R-1$, and $k=0, \ldots, N-1$ are the time frame and frequency bin indices, respectively.

Let $\mathbf{Z}$ be an $N \times R$ measurement matrix corresponding to the STFT magnitudesquare of the underlying signal $\mathbf{x}$.

$$
\mathbf{Z}=|\mathbf{X}|^{2}
$$

The aim of the algorithms in [13] is to estimate $\mathbf{x}$ from $\mathbf{Z}$. The key idea is to introduce redundancy in the magnitude-only measurements by maintaining a substantial overlap between adjacent short-time sections [1]]. For sufficiently long window length, the solution can be obtained via a LS method. When these conditions are not met, a 
45 GD algorithm is used to solve the phase retrieval problem. Experiments show that the algorithms in [13] can exactly recover $\mathbf{x}$ from $\mathbf{Z}$.

The algorithm in [13] begins by taking the DFT of the STFT measurement (2I), as follows

$$
\begin{aligned}
Y(\tau, l) & =\frac{1}{N} \sum_{k=0}^{N-1} Z(\tau, k) e^{-2 j \pi k l / N} \\
& =\sum_{n=0}^{N-1} x(n) x^{*}(n+l) g(\tau L-n) g(\tau L-n-l),
\end{aligned}
$$

where $Y(\tau, l)$ is equal to zero for all $\tau$ when $W \leq l \leq(N-W)$ and can be interpreted as a " $W$ bandlimited" function. The DFT is normalized by $1 / N$. For a fixed $\tau, \mathbf{Y}$ can be seen as the autocorrelation of $\mathbf{x} \circ \mathbf{g}_{\tau L}$, where $\mathbf{g}_{\tau L}=\{g(\tau L-n)\}_{n=0}^{N-1}$. This step simplifies the structure of the data and leads almost directly to the uniqueness results (see [133] for more details).

For $W \geq\lceil(N+1) / 2\rceil$ and $L=1$, the problem of recovering $\mathbf{x}$ from the measurement $\mathbf{Z}$ can be posed equivalently as a constrained LS problem derived from (B).

$$
\begin{aligned}
& \min _{\mathbf{x} \in \mathbb{C}^{N}} \sum_{l=-(W-1)}^{W-1}\left\|\mathbf{y}_{l}-\mathbf{G}_{l} \operatorname{diag}(\mathbf{X}, l)\right\|_{2}^{2} \\
& \text { subject to } \mathbf{X}=\mathbf{x x}^{*},
\end{aligned}
$$

where $\mathbf{y}_{l}=\{Y(\tau, l)\}_{\tau=0}^{R-1}$, and the $(\tau, n)$ th entry of the matrix $\mathbf{G}_{l} \in \mathbb{R}^{R \times N}$ is given by $g(\tau L-n) g(\tau L-n-l)$. The circulant matrix $\mathbf{G}_{l}$ can be factored as $\mathbf{G}_{l}=\mathbf{F}^{*} \sum_{l} \mathbf{F}$, where $\mathbf{F}$ is the DFT matrix and $\sum_{l}$ is a diagonal matrix (as in [II3]). For long enough windows, 55 [13] shows that the LS algorithm is effective for recovering $\mathbf{x}$ from $\mathbf{Z}$.

For $2 \leq W \leq\lceil N / 2\rceil$, let $\mathbf{D}_{\tau L} \in \mathbb{R}^{N \times N}$ be a diagonal matrix composed of the entries of $\mathbf{g}_{\tau L}$, then a non-convex loss function formed from (B]) can be used for recovering $\mathbf{x}$ from $\mathbf{Z}$ [113] , as follows

$$
f(\mathbf{x})=\frac{1}{2} \sum_{\tau=0}^{R-1} \sum_{l=-(W-1)}^{W-1}\left(\mathbf{x}^{*} \mathbf{H}_{\tau, l} \mathbf{x}-Y(\tau, l)\right)^{2},
$$

where $\mathbf{H}_{\tau, l}=\mathrm{P}_{-l} \mathbf{D}_{\tau L} \mathbf{D}_{\tau L-l}, \mathbf{x}^{*} \mathbf{H}_{\tau, l} \mathbf{x}=\operatorname{tr}\left(\mathbf{X H}_{\tau, l}\right), \mathrm{P}_{-l}=\mathrm{P}_{l}^{T},\left(\mathrm{P}_{l} x\right)(n)=x(n+l)$, and $R$ is defined as in (II).

A GD algorithm can be adopted to minimize (5). It was shown in [1]3] that, if $\mathbf{g}$ is not long enough, the GD algorithm is effective for recovering $\mathbf{x}$ from $\mathbf{Z}$.

Initialization: Initialization is important to the GD algorithm. If $L=1$, the initialization of $\mathbf{x}_{0}$ is determined by (更) directly. In the case of $L>1, \mathbf{y}_{l}=\{Y(\tau, l)\}_{\tau=0}^{R-1}$ has some missing entries. The up-sampled version $\tilde{\mathbf{y}}_{l}$ is obtained by expansion and 
interpolation,

$$
\begin{aligned}
& Y(n, l)= \begin{cases}Y(\tau, l), & n=\tau L, \\
0, & \text { otherwise, }\end{cases} \\
& \text { Denote } \hat{\mathbf{y}}_{l}=\{Y(n, l)\}_{n=0}^{N-1} \text { for fixed } l, \text { we have } \\
& \tilde{\mathbf{y}}_{l}=\left(\mathbf{F}_{p}^{*} \mathbf{F}_{p}\right) \hat{\mathbf{y}}_{l},
\end{aligned}
$$

60 where $\mathbf{F}_{p}$ is a partial Fourier matrix consisting of the first $R$ rows of the DFT matrix $\mathbf{F}$ defined as in (更). Then the initialization $\mathbf{x}_{0}$ can be obtained by (田).

\section{Proposed Model and Assumptions}

The aim here is to recover multiple underlying sources from their mixed STFT magnitude-square measurements coupled with noise. In this section, a mathematical model, the assumptions and an algorithm are presented for the problem of multi-source phase retrieval.

\subsection{The proposed model for multi-source phase retrieval}

As is shown in Fig. W, the multiple underlying sources are denoted as $\mathbf{S}=\left\{\mathbf{s}_{1}, \mathbf{s}_{2}, \ldots, \mathbf{s}_{M}\right\} \in$ $\mathbb{C}^{M \times N}$. The mixed signals are defined as $\mathbf{X}=\mathbf{A S}$, where $\mathbf{X}=\left\{\mathbf{x}_{1}, \mathbf{x}_{2}, \ldots, \mathbf{x}_{M}\right\} \in \mathbb{C}^{M \times N}$

70 and the mixing matrix $\mathbf{A} \in \mathbb{R}^{M \times M}$.

Denote $\mathbf{F}=\left\{\mathbf{X}_{1}, \mathbf{X}_{2}, \ldots, \mathbf{X}_{M}\right\}$ as the STFT matrices of $\mathbf{X}=\left\{\mathbf{x}_{1}, \mathbf{x}_{2}, \ldots, \mathbf{x}_{M}\right\}$, where $\mathbf{X}_{i} \in \mathbb{C}^{R \times N}$. The elements of $\mathbf{F}$ can be defined as

$$
\left\{\begin{array}{c}
X_{1}(\tau, k)=\sum_{n=0}^{N-1} x_{1}(n) g(\tau L-n) e^{-2 j \pi k n / N}, \\
\vdots \\
X_{M}(\tau, k)=\sum_{n=0}^{N-1} x_{M}(n) g(\tau L-n) e^{-2 j \pi k n / N},
\end{array}\right.
$$

where $\mathbf{g}, \tau, k, L, R$ and STFT $\mathbf{X}_{i}$ are similar to those in (II).

Denote the STFT magnitude-squared measurements as $|\mathbf{F}|^{2}=\left\{\left|\mathbf{X}_{1}\right|^{2}, \ldots,\left|\mathbf{X}_{M}\right|^{2}\right\}$. We have the following signal model

$$
\mathbf{Z}_{i}=\left|\mathbf{X}_{i}\right|^{2}+\mathbf{N}_{i}
$$

where $i=1, \ldots, M$ and $\mathbf{N}_{i}$ is a random $R \times N$ matrix which represents noise. Thus $\mathbf{Z}=\left\{\mathbf{Z}_{1}, \ldots, \mathbf{Z}_{M}\right\}$ and $\mathbf{N}=\left\{\mathbf{N}_{1}, \ldots, \mathbf{N}_{M}\right\}$.

The aim of multi-source phase retrieval is to recover the phases of the underlying sources $\mathbf{S}$ from the phaseless STFT measurements $\mathbf{Z}$ corrupted by noise $\mathbf{N}$. To address this problem, two assumptions and a two-step algorithm are proposed as discussed next. 


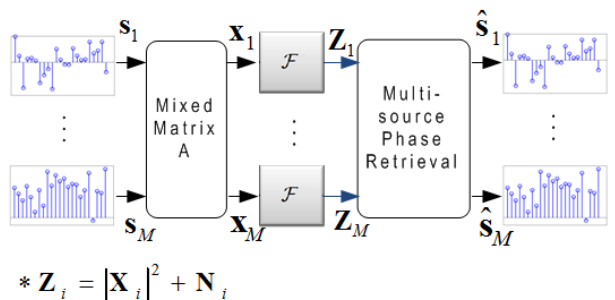

Figure 1: The proposed model for multi-source phase retrieval.

\subsection{Assumptions for multi-source phase retrieval}

Two assumptions are utilized for constructing the multi-source phase retrieval model:

(1) The mixed signals $\mathbf{X}$ are linear mixtures of the multiple underlying source signals $\mathbf{S}$ multiplied by the mixing matrix $\mathbf{A}$.

(2) The multiple underlying sources $\mathbf{S}$ are statistically independent.

\section{Proposed RMSPR Algorithm for Multi-Source Phase Retrieval}

The proposed method is composed of two steps. The first step is to recover the mixed signals $\mathbf{X}$ from their mixed phaseless STFT measurements $\mathbf{Z}$. The second step is to recover the underlying source signals $\mathbf{S}$ from the mixed signals $\mathbf{X}$.

In order to obtain acquired data, we take DFT for each $\mathbf{Z}$. Take $\mathbf{Z}_{i}$ as an example, the DFT of the $i$ th measurement $Y_{i}(\tau, l)$ can be described by

$$
\begin{aligned}
Y_{i}(\tau, l) & =\frac{1}{N} \sum_{k=0}^{N-1} Z_{i}(\tau, k) e^{-2 j \pi k l / N} \\
& =\sum_{n=0}^{N-1} x_{i}(n) x_{i}^{*}(n+l) g(\tau L-n) g(\tau L-n-l),
\end{aligned}
$$

where $\mathbf{g}_{\tau L}$ and $Y_{i}(\tau, l)$ are defined as in (B)).

For $2 \leq W \leq\lceil N / 2\rceil$, a GD algorithm is adopted to recover the $i$ th mixed signal by minimizing the non-convex loss function [113],

$$
f\left(\mathbf{x}_{i}\right)=\frac{1}{2} \sum_{\tau=0}^{R-1} \sum_{l=-(W-1)}^{W-1}\left(\mathbf{x}_{i}^{*} \mathbf{H}_{\tau, l} \mathbf{x}_{i}-Y_{i}(\tau, l)\right)^{2},
$$

where $\mathbf{H}_{\tau, l}$ and $\mathbf{x}_{i}^{*} \mathbf{H}_{\tau, l} \mathbf{x}_{i}$ are defined as in (Б).

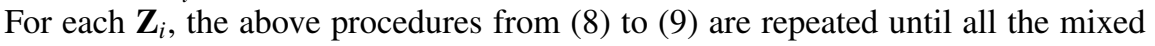
signals $\mathbf{X}$ have been recovered. To remove the magnitude effect of the mixing matrix, the recovered mixed signals are normalized as $\hat{\mathbf{X}}=\left\{\hat{\mathbf{x}}_{1}, \hat{\mathbf{x}}_{2}, \ldots, \hat{\mathbf{x}}_{M}\right\}$.

All the normalized mixed signals $\hat{\mathbf{X}}$ are used as inputs to an independent component analysis algorithm [16], such as the Joint Approximative Diagonalization of Eigenmatrix (JADE) algorithm for complex-valued signals [177]. 
More specifically, the mixed signals are whitened as $\tilde{\mathbf{X}}=\mathbf{U} \hat{\mathbf{X}}$ using a whitening matrix $\mathbf{U}$ [17].

A maximal set of cumulant matrices $\left\{\mathbf{Q}_{i}^{\tilde{\mathbf{X}}}\right\}$ is formed

$$
\begin{aligned}
& \mathbf{Q}_{i}^{\tilde{\mathbf{X}}}=\mathbf{W G}\left(\mathbf{M}_{i}\right) \mathbf{W}^{\dagger} \\
& \mathbf{G}\left(\mathbf{M}_{i}\right)=\operatorname{diag}\left[\mathrm{k}\left(\mathbf{s}_{1}\right) \mathbf{w}_{1}^{\dagger} \mathbf{M}_{i} \mathbf{w}_{1}, \ldots, \mathrm{k}\left(\mathbf{s}_{M}\right) \mathbf{w}_{M}^{\dagger} \mathbf{M}_{i} \mathbf{w}_{M}\right],
\end{aligned}
$$

where $\mathbf{w}_{k}$ denotes the $k$ th column of $\mathbf{W}$, which diagonalizes $\mathbf{G}\left(\mathbf{M}_{i}\right)$ for any matrix $\mathbf{M}_{i}$, and $\mathrm{k}\left(\mathbf{s}_{i}\right)$ means the kurtosis of $\mathbf{s}_{i}$ [17].

A rotation matrix $\hat{\mathbf{V}}$ is obtained by enforcing the off-diagonal elements of the cumulant matrices as close to zero as possible [16].

$$
\hat{\mathbf{V}}=\operatorname{argmin} \sum_{i} \operatorname{Off}\left(\mathbf{V}^{\dagger} \mathbf{Q}_{i}^{\tilde{\mathbf{X}}} \mathbf{V}\right) .
$$

The mixing matrix is then estimated as $\mathbf{A}=\hat{\mathbf{V}} \mathbf{U}^{-1}$. The underlying sources are recovered as $\mathbf{S}=\mathbf{A}^{-1} \hat{\mathbf{X}}$, and then normalized as $\hat{\mathbf{S}}=\left\{\hat{\mathbf{s}}_{1}, \hat{\mathbf{s}}_{2}, \ldots, \hat{\mathbf{s}}_{M}\right\}$. The proposed algorithm is summarized in Algorithm $\mathbf{W}$.

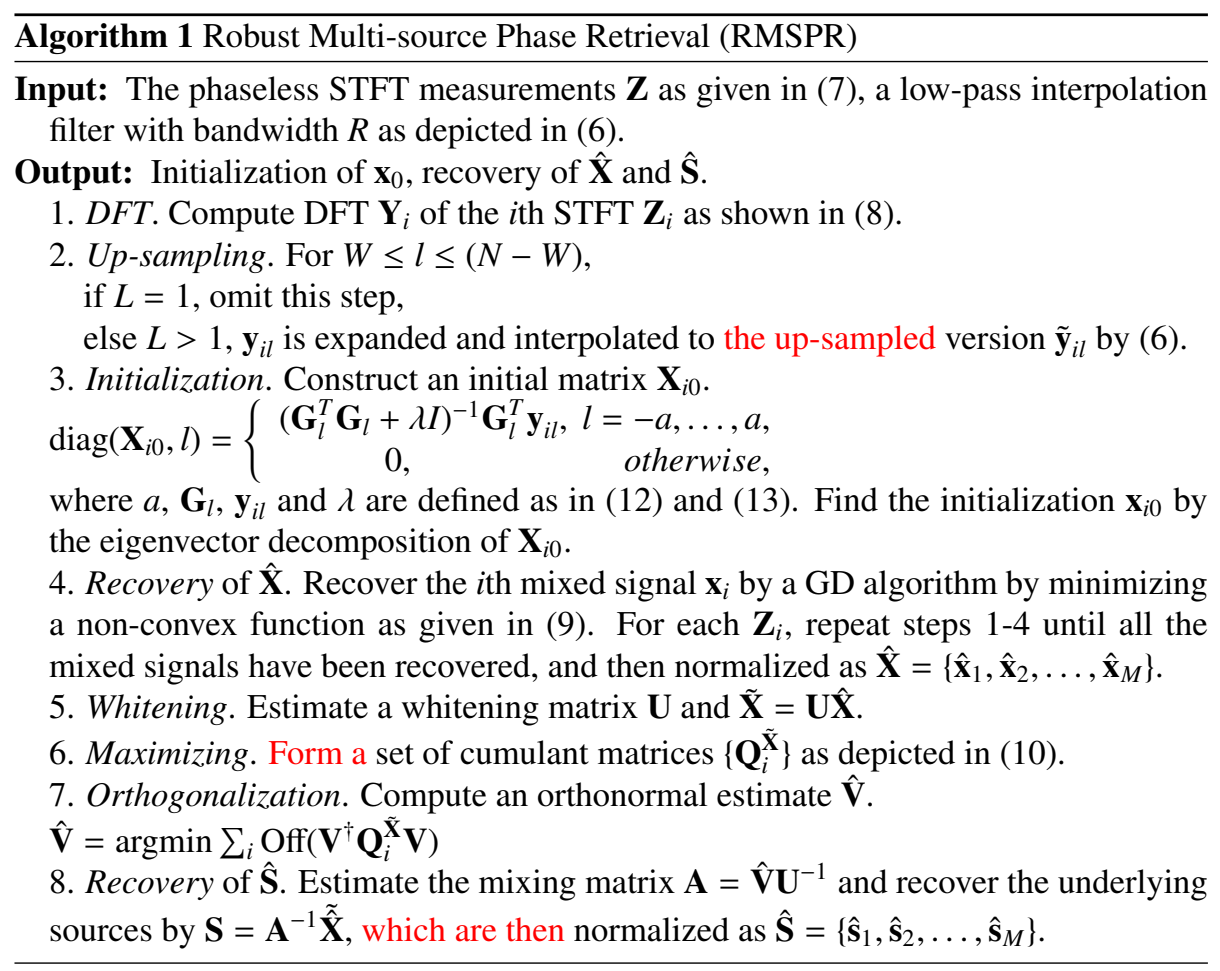

Improved loss function for initialization: It is shown in [13] that (B)) can be posed equivalently as a constrained LS problem as (田). With (田), it is prone to over-fitting with less data, and requires the rank restriction of $\mathbf{G}_{l}$. To address this issue, a penalty 
term is introduced as follows, for $L=1$,

$$
\begin{aligned}
& \min _{\mathbf{x}_{i} \in \mathbb{C}^{N}} \sum_{l=-(W-1)}^{W-1}\left\|\mathbf{y}_{i l}-\mathbf{G}_{l} \operatorname{diag}\left(\mathbf{X}_{i}, l\right)\right\|_{2}^{2}+\lambda\left\|\operatorname{diag}\left(\mathbf{X}_{i}, l\right)\right\|_{2}^{2} \\
& \text { subject to } \mathbf{X}_{i}=\mathbf{x}_{i} \mathbf{x}_{i}^{*},
\end{aligned}
$$

where $\mathbf{G}_{l}$ and $\mathbf{y}_{i l}$ are defined as in (四), and $\lambda$ is a regularization coefficient. The modification of (田) may provide significant benefits in two aspects. First, it prevents overfitting and improves generalization performance. Second, it relaxes the rank restriction of $\mathbf{G}_{l}$.

The initialization of the GD algorithm is obtained by the principal eigenvector of a designed matrix which is constructed as the solution to (피). The designed matrix is given as

$$
\operatorname{diag}\left(\mathbf{X}_{i 0}, l\right)=\left\{\begin{array}{lr}
\left(\mathbf{G}_{l}^{T} \mathbf{G}_{l}+\lambda \mathbf{I}\right)^{-1} \mathbf{G}_{l}^{T} \mathbf{y}_{i l}, l=-a, \ldots, a, \\
0, & \text { otherwise } .
\end{array}\right.
$$

Then the initialization $\mathbf{x}_{i 0}$ of the proposed GD algorithm can be constructed by $\mathbf{X}_{i 0}$.

For $L>1$, the up-sampled version $\tilde{\mathbf{y}}_{i l}$ is obtained from $\mathbf{y}_{i l}=\left\{Y_{i}(\tau, l)\right\}_{\tau=0}^{R-1}$ by (6). Then the initialization $\mathbf{x}_{i 0}$ is estimated by ([13).

\section{Numerical Experiments}

In this section, we conduct numerical simulations to demonstrate the competitive performance of the proposed RMSPR algorithm.

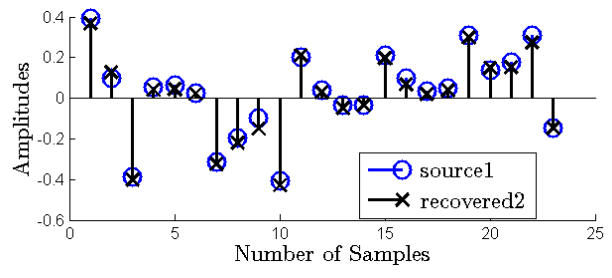

(a) Recovery of the underlying source $\hat{\mathbf{s}}_{1}$

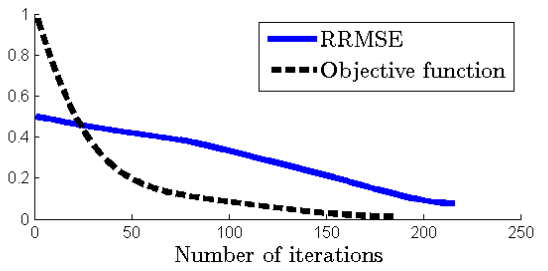

(c) RRMSE and the objective function values of $\hat{\mathbf{x}}_{1}$

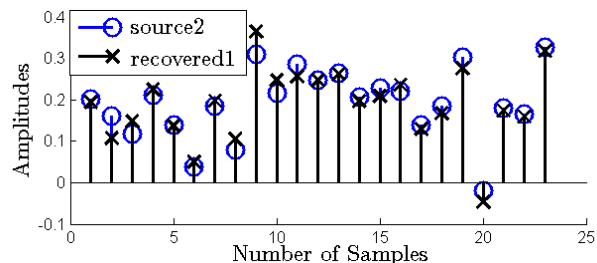

(b) Recovery of the underlying source $\hat{\mathbf{s}}_{2}$

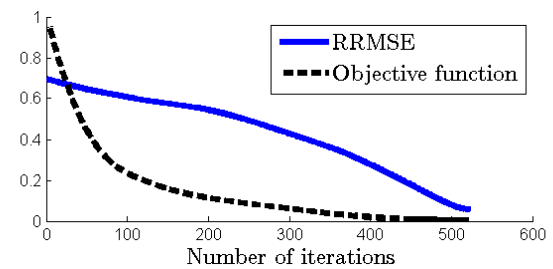

(d) RRMSE and the objective function values of $\hat{\mathbf{x}}_{2}$

Figure 2: Recovery of the underlying sources (length $N=23$ ) using a rectangular window (with the level of noise at $\mathrm{SNR}=25 \mathrm{~dB}$ ). 
We consider the case of $M=2$. The underlying sources are drawn randomly and statistically independent. The elements of the mixing matrix are drawn randomly with zero mean and unit variance. The mixed measurements are corrupted by additive Gaussian noise with zero mean and unit variance, at the level from $5 \mathrm{~dB}$ to $25 \mathrm{~dB}$. The performance of the proposed algorithm can be evaluated by the relative root mean squared error (RRMSE) and the signal to noise ratio (SNR), where RRMSE is defined as follows

$$
\operatorname{RRMSE}=\frac{\operatorname{RMS}\left(\mathbf{s}_{i}-\hat{\mathbf{s}}_{i}\right)}{\operatorname{RMS}\left(\mathbf{s}_{i}\right)}, \quad i=1, \ldots, M .
$$

The first experiment examines the recovery quality of the proposed RMSPR algorithm for the maximal overlapping between adjacent windows $(L=2)$. If $L$ is very large, $\mathbf{y}_{l}$ will have many missing entries and the underlying signals cannot be recovered ( $N=23$ ), the noise level at $25 \mathrm{~dB}$, and the number of short-time windows is $R=\lceil N / L\rceil$. The algorithm is prone to over-fitting with a large regularization coefficient $\lambda$, and converges slowly with a small step size $\mu$. Therefore, $\lambda, \mu$, and the statistical threshold for stopping joint diagonalization, are set to be $0.01,0.005$, and $1 / 100 \sqrt{N}$ [ए7], respectively. As shown in Fig. 口, the final RRMSEs of the recovered signals are less than 0.1 which demonstrate the effectiveness of the proposed algorithm for phase recovery of multiple sources.

The second experiment is to evaluate the performance of the RMSPR algorithm for the signal length $(N=43)$ with respect to the length of the maximal overlapping 125 between adjacent windows $L$. All the other parameters were set identical to those in the first experiment. Fig. B presents the average RRMSE for 45 experiments of the recovered mixed signals $\hat{\mathbf{X}}=\left\{\hat{\mathbf{x}}_{1}, \hat{\mathbf{x}}_{2}\right\}$ and the recovered underlying sources $\hat{\mathbf{S}}=\left\{\hat{\mathbf{s}}_{1}, \hat{\mathbf{s}}_{2}\right\}$ for different $L$. For low values of $L=1,2$, the RRMSEs of $\hat{\mathbf{X}}$ and $\hat{\mathbf{S}}$ are relatively low (at around 0.2 and 0.1 respectively), and in this case, the underlying sources are well 30 recovered. For high values of $L=3,4,5$, the RRMSE of $\hat{\mathbf{X}}$ is smaller than that of $\hat{\mathbf{S}}$ and the values are mostly above 0.4 . These experimental results indicate that the interpolation is effective in up-sampling measurements especially for low values of $L$. The proposed algorithm has better performance in recovering $\hat{\mathbf{S}}$ than $\hat{\mathbf{X}}$ only for low values of $L$.

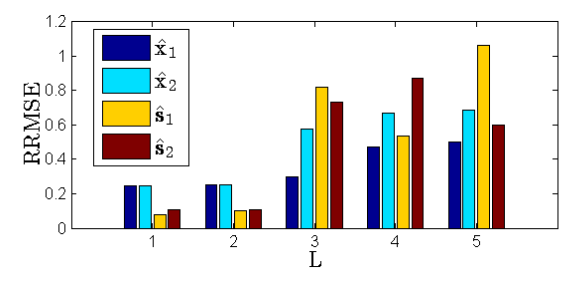

Figure 3: The average RRMSE for 45 experiments of the recovered mixed signals $\hat{\mathbf{X}}$ and the recovered underlying sources $\hat{\mathbf{S}}$ for different $L$.

The third experiment conducted is to evaluate the performance of the RMSPR algorithm with respect to different SNRs for the signal length $(N=43)$, with other parameters set as those in the previous experiments. Fig. 田 demonstrates the aver- 
age RRMSE for 45 experiments of the recovered mixed signals $\hat{\mathbf{X}}=\left\{\hat{\mathbf{x}}_{1}, \hat{\mathbf{x}}_{2}\right\}$ and the recovered underlying sources $\hat{\mathbf{S}}=\left\{\hat{\mathbf{s}}_{1}, \hat{\mathbf{s}}_{2}\right\}$ for different SNRs. With the increase in $140 \mathrm{SNR}$, the RRMSE of $\hat{\mathbf{X}}$ decreases slightly whereas the RRMSE of $\hat{\mathbf{S}}$ drops rapidly. For $\mathrm{SNR}=15 \mathrm{~dB}, 20 \mathrm{~dB}, 25 \mathrm{~dB}$, the RRMSEs of $\hat{\mathbf{S}}$ are relatively low and less than 0.15 . The results mean that the RMSPR algorithm has better anti-noise performance in $\hat{\mathbf{S}}$ than $\hat{\mathbf{X}}$ especially for high values of SNR.

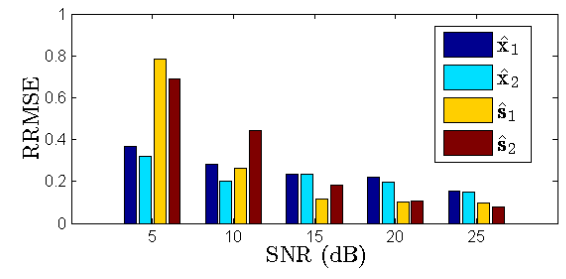

Figure 4: The average RRMSE for 45 experiments of the recovered mixed signals $\hat{\mathbf{X}}$ and the recovered underlying sources $\hat{\mathbf{S}}$ for different SNRs.

\section{Conclusion}

145

We have presented a new model and algorithm for recovering multiple underlying sources from their mixed multi-channel STFT magnitude-square measurements. The proposed RMSPR algorithm couples a GD algorithm by minimizing a non-convex loss function with an ICA algorithm. An improved LS loss function is also presented to find the initialization of the GD algorithm. Numerical experiments show that the proposed algorithm performs well in estimating the phase of multiple sources and the mixing information. For low values of $L$ (e.g. $L=1,2$ ) and high values of SNR (e.g. $15 \mathrm{~dB}$, $20 \mathrm{~dB}$, and $25 \mathrm{~dB}$ ), the final RRMSEs of the recovered signals are relatively low and less than 0.15 .

In future, it is interesting to investigate how to incorporate conditions such as window length, mix-mode, or maximal overlapping between adjacent windows into the multi-source phase retrieval algorithm.

\section{Acknowledgements}

The authors wish to thank the handling editor and the anonymous reviewers for their detailed suggestions and corrections. This work was supported by the National Natural Science Foundation of China under Grant 61301250, Young Academic Leaders of Higher Learning Institutions of Shanxi Province Office of Education under Grant [2015]3, and Shanxi Scholarship Council of China under Grant 2014-060. The work was conducted during Guo's visit at the University of Surrey. 


\section{References}

165 [1] J. Kulmer, P. Mowlaee, Phase estimation in single channel speech enhancement using phase decomposition, IEEE Signal Processing Letters 22 (5) (2015) 598602.

[2] A. Ahmed, B. Recht, J. Romberg, Blind deconvolution using convex programming, IEEE Transactions on Information Theory 60 (3) (2014) 1711-1732.

170 [3] J. Miao, T. Ishikawa, I. K. Robinson, M. M. Murnane, Beyond crystallography: Diffractive imaging using coherent X-ray light sources, Science 348 (6234) (2015) $530-535$.

[4] Y. Shechtman, Y. C. Eldar, O. Cohen, H. N. Chapman, J. Miao, M. Segev, Phase retrieval with application to optical imaging: A contemporary overview, IEEE Signal Processing Magazine 32 (3) (2015) 87-109.

[5] L. K. Patton, B. D. Rigling, Phase retrieval for radar waveform optimization, IEEE Transactions on Aerospace and Electronic Systems 48 (4) (2012) 3287 3302.

[6] J. R. Fienup, Phase retrieval algorithms: A comparison, Applied Optics 21 (15) (1982) 2758-2769.

[7] R. W. Gerchberg, A practical algorithm for the determination of the phase from image and diffraction plane pictures, Optik 35 (1972) 237-246.

[8] K. Huang, Y. C. Eldar, N. D. Sidiropoulos, Phase retrieval from 1D fourier measurements: Convexity, uniqueness, and algorithms, IEEE Transactions on Signal Processing 64 (23) (2016) 6105-6117.

[9] D. Griffin, J. Lim, Signal estimation from modified short-time Fourier transform, IEEE Transactions on Acoustics, Speech, and Signal Processing 32 (2) (1984) 236-243.

[10] E. J. Candes, Y. C. Eldar, T. Strohmer, V. Voroninski, Phase retrieval via matrix completion, SIAM review 57 (2) (2015) 225-251.

[11] Y. C. Eldar, P. Sidorenko, D. G. Mixon, S. Barel, O. Cohen, Sparse phase retrieval from short-time Fourier measurements, IEEE Signal Processing Letters 22 (5) (2015) 638-642.

[12] K. Jaganathan, Y. C. Eldar, B. Hassibi, STFT phase retrieval: Uniqueness guarantees and recovery algorithms, IEEE Journal of Selected Topics in Signal Processing 10 (4) (2016) 770-781.

[13] T. Bendory, Y. C. Eldar, Non-convex phase retrieval from STFT measurements, arXiv preprint arXiv:1607.08218. 
[14] J. I. Echeveste, M. Á. G. de Aza, J. Rubio, J. Zapata, Near-optimal shaped-beam synthesis of real and coupled antenna arrays via 3-D-FEM and phase retrieval, IEEE Transactions on Antennas and Propagation 64 (6) (2016) 2189-2196.

[15] K. Jaganathan, Y. C. Eldar, B. Hassibi, Phase retrieval: An overview of recent developments, arXiv preprint arXiv:1510.07713.

[16] A. Hyvärinen, J. Karhunen, E. Oja, Independent component analysis, Vol. 46, John Wiley \& Sons, 2004.

[17] J. F. Cardoso, High-order contrasts for independent component analysis, Neural Computation 11 (1) (1999) 157-192. 